\title{
The canadian traveller problem and its competitive analysis
}

\author{
Yinfeng Xu • Maolin Hu • Bing Su • Binhai Zhu • \\ Zhijun Zhu
}

Published online: 9 April 2008

(C) The Author(s) 2008. This article is published with open access at Springerlink.com

\begin{abstract}
From the online point of view, we study the Canadian Traveller Problem (CTP), in which the traveller knows in advance the structure of the graph and the costs of all edges. However, some edges may fail and the traveller only observes that upon reaching an adjacent vertex of the blocked edge. The goal is to find the leastcost route from the source $O$ to the destination $D$, more precisely, to find an adaptive strategy minimizing the competitive ratio, which compares the performance of this strategy with that of a hypothetical offline algorithm that knows the entire topology in advance. In this paper, we present two adaptive strategies - a greedy or myopic strategy and a comparison strategy combining the greedy strategy and the reposition strategy in which the traveller backtracks to the source every time when he/she sees a failed edge. We prove tight competitive ratios of $2^{k+1}-1$ and $2 k+1$ respectively
\end{abstract}

We would like to acknowledge the support from NSF of China (No. 70525004, No. 70121001 and No. 60736027), and the support from K.C. Wong Education Foundation, Hong Kong.

Y. Xu · B. Su $(\bowtie) \cdot Z$. Zhu

School of Management, Xi' an Jiaotong University, Xi' an 710049, China

e-mail: subing@mail.xjtu.edu.cn

Y. Xu

e-mail: yfxu@mail.xjtu.edu.cn

Z. Zhu

e-mail: zhuzhj@ctbri.com.cn

Y. Xu $\cdot$ B. Su

The State Key Lab for Manufacturing Systems Engineering, Xi'an 710049, China

M. Hu

Department of Mathematics, Guyuan Teachers College, Guyuan 756000, China

e-mail: humaolin2000@163.com

B. Zhu

Department of Computer Science, Montana State University, Bozeman, MT 59717, USA

e-mail: bhz@esus.cs.montana.edu 
for the two strategies, where $k$ is the number of failed edges in the graph. Finally, we propose an explanation of why the greedy strategy and the comparison strategy are usually preferred by drivers in an urban traffic environment, based on an argument related to the length of the second-shortest path in a grid graph.

Keywords Online algorithms · Competitive analysis · Greedy strategy · Comparison strategy

\section{Introduction}

The Canadian Traveller Problem (CTP) has been introduced in Papadimitriou and Yannakakis (1991) and is defined as follows: Suppose that we have a graph and a traveller has to travel from the source $O$ to reach the destination $D$, and the traveller knows in advance the structure of the graph and the costs of all the edges. However, some edges may fail and the traveller only observes that upon reaching an adjacent vertex of the blocked/failed edge. The problem is to devise a good travel strategy from $O$ to $D$ based on this partial information, with no knowledge of future edge blockage. Under this setting, Papadimitriou and Yannakakis proved that devising an online algorithm with a bounded competitive ratio is PSPACE-complete (Papadimitriou and Yannakakis 1991). Several variations of the CTP were studied since, for example in Bar-Noy and Schieber (1991). If there is a given parameter which bounds the number of blocked edges from above, the resulting problem is called the $k$-Canadian Traveller Problem ( $k$-CTP). Bar-Noy and Schieber studied the $k$-CTP, but they did not consider the problem from a competitive analysis point of view (Bar-Noy and Schieber 1991). Instead, they consider the worst-case criterion which aims at a strategy where the maximum cost is minimized. Westphal (2008) considered the online version of $k$-CTP and gave an reposition strategy in which the traveller backtracks to the source every time when he/she sees a failed edge and then follows the optimal shortest path in the graph (with the known blocked edges removed). He showed that no deterministic online algorithm can achieve a competitive ratio smaller than $2 k+1$. The same bound was in fact obtained by us independently (Zhu et al. 2003; $\mathrm{Hu}$ 2005). Westphal also showed that randomization can not improve the competitive ratio substantially. He showed that by establishing a lower bound of $k+1$ for the competitiveness of any randomized online algorithm against an oblivious adversary (Westphal 2008). For the Recoverable CTP, in which an upper bound on the number of blockages is known in advance and the recovery times are not very long compared with the travel times, Bar-Noy and Schieber presented a polynomial-time travel strategy which guarantees the shortest worst-case travel time. For the Stochastic Recoverable CTP, again when the recovery times are not very long relative to the travel times, they also presented a polynomial-time strategy which minimizes the expected travel time (Bar-Noy and Schieber 1991). Finally, they considered the "dual" problem of the CTP known as the $k$-Vital Edges Problem, and proved that it is NP-hard even when the travel time along all the roads is a constant (Bar-Noy and Schieber 1991).

The Canadian Traveller Problem and its variations can be viewed as an abstraction for the online shortest paths/routing problems. The online shortest paths/routing 
problem is defined as follows: The traveller knows a graph and two different vertices $O$ and $D$. At each step, the traveller may choose a path from $O$ to $D$. More specifically, at each time step $t$, the traveller picks a path from $O$ to $D$, and simultaneously an adversary (or the nature) chooses a set of edge costs. The edge costs are then revealed and the traveller pays the cost for his/her selected path. For the online shortest paths/routing problem, Takimoto and Warmuth designed an efficient algorithm with a nice structure to mimic the distribution of all paths that would be chosen by some exponential-time algorithm, but with efficient implicit calculations (Takimoto and Warmuth 2003). Kalai and Vempala presented some follow-the-leader style algorithms which perform nearly as well as the best single decision, where the best is chosen with the benefit of hindsight (Kalai and Vempala 2005). Blum, Even-Dar and Ligett showed that in the Wardrop setting of multicommodity flow and infinitesimal agents, the behavior will approach Nash equilibrium at a rate that depends polynomially on the travellers' regret bounds and the maximum slope of any latency function (Blum et al. 2006).

In this paper, from the online point of view, we study the CTP with failed edges for general networks. Our goal is to find the least-cost route from the source $O$ to the destination $D$, more precisely, to find an adaptive strategy minimizing the competitive ratio, which compares the performance of this strategy with that of a hypothetical offline algorithm that knows the entire topology in advance. We present some competitive ratio that is related to the number of blockages, but irrelevant to the location of blockages. While the reposition strategy has been shown to be optimal (in the worst case sense), it is not practical and is against human nature under many situations. (Recall that in the reposition strategy the traveller backtracks to the source every time when he/she sees a failed edge.) So we propose two adaptive strategies - a greedy or myopic strategy and a comparison strategy combining the greedy strategy and the reposition strategy. By a competitive analysis, we prove that their optimal competitive ratios are $2^{k+1}-1$ and $2 k+1$ respectively, where $k$ is the total number of blockages. When the network graph is a special one (say, close to a grid), it turns out that the performance measure of the greedy strategy is better than that of the reposition strategy. This offers a reasonable explanation why drivers prefer the greedy or the comparison strategy to the reposition strategy in an urban traffic environment, etc.

The organization of this paper is as follows. In Sect. 2, the problem definition and some assumptions which we will use are briefly reviewed. In Sect. 3 we propose and investigate two online strategies for the CTP. In Sect. 4, we consider the performance measure of the strategies in a special network which closely models an urban traffic environment.

\section{Problem statement and formulation}

Let $G=(V, E)$ be an undirected network with $|V|=n$ vertices and $|E|=m$ edges. Let $O$ denote the source and $D$ the destination, and let $w(e)$ denote a nonnegative real weight associated to each edge $e$. Let $E_{k}=\left(e_{1}, e_{2}, \ldots, e_{i}, \ldots, e_{k}\right)$ denote the blocked edge sequence, where $e_{i}=\left(O_{i}, Y_{i}\right)$. Suppose that the traveller has to reach $D$ starting from $O$, then the problem is to design a good travel strategy based on the 
partial information, with no knowledge of future blockages. A natural question is: What is the difference of the performance measure of different strategies?

In order to discuss this question, we make the following assumptions.

\section{Basic Assumptions:}

- The traveller knows the entire graph and edge costs in advance.

- Some edges may fail and the traveller does not know which one will fail in advance.

- The traveller sees that an edge $e_{i}=\left(O_{i}, Y_{i}\right)$ is blocked upon arriving at $O_{i}$.

- $G$ is still connected even if the blocked edges are removed.

- Blockages happen at some of the edges. Once an edge is blocked, it remains blocked forever.

If all of the failed edges are known in advance, then the problem becomes an offline problem, and the optimal travel strategy is obtained by following the shortest path from $O$ to $D$ after blockages are removed from the graph. If the blocked edges are unpredictable, then the problem is obviously an online problem.

For the classical online problem (Sleator and Tarjan 1985; Borodin and El-Yaniv 1998; Fiat et al. 1998; Fiat and Woeginger 1998), the competitive ratio is a value irrelevant to the sequences of events and is formally defined as follows.

Let $C_{\text {opt }}(R)$ denote the cost of the optimal offline problem $R, C_{A}(R)$ denote the cost of the strategy $A$ for the corresponding online problem. Strategy $A$ is said to be $\alpha$-competitive if

$$
C_{A}(R) \leq \alpha \cdot C_{o p t}(R)+\beta
$$

holds, where $\alpha$ and $\beta$ are constants not related to $R$.

For the CTP, we give a slightly new definition for the competitive ratio. Let $C_{\text {opt }}\left(O D \mid E_{k}\right)$ be the cost of the optimal offline shortest path from the source $O$ to the destination $D$, after blocked edges $E_{k}$ are removed from the graph, and let $C_{A}\left(O D \mid E_{k}\right)$ be the corresponding cost of the online strategy $A$ for the traveller to go from $O$ to $D$.

Strategy $A$ is called $c(k)$-competitive, if the following inequality holds:

$$
C_{A}\left(O D \mid E_{k}\right) \leq c(k) \cdot C_{o p t}\left(O D \mid E_{k}\right),
$$

where $c(k)$ is related to $k$ and irrelevant to the location of blockages.

Let $C_{\text {opt }}(O D)$ be the optimal offline shortest path cost without any blockage in $G$. The following lemma can be easily obtained.

Lemma 1 It holds that

$$
\begin{aligned}
C_{o p t}(O D) & \leq C_{o p t}\left(O D \mid E_{1}\right) \leq C_{o p t}\left(O D \mid E_{2}\right) \\
& \leq \cdots \leq C_{o p t}\left(O D \mid E_{k-1}\right) \leq C_{o p t}\left(O D \mid E_{k}\right)
\end{aligned}
$$

\section{Competitive analysis of two online strategies}

In this section, we present two online strategies for the CTP and analyze their corresponding competitive ratios. As discussed earlier, the reposition strategy, while being 
optimal in the worst case, is not a natural solution in practice. So it makes sense to present some more natural solutions.

\subsection{The greedy strategy}

Greedy Strategy: When the traveller reaches $O_{i}$ and knows that the edge $e_{i}=$ $\left(O_{i}, Y_{i}\right)$ is blocked, he/she takes the shortest path from $O_{i}$ to $D$ which does not make use of $e_{i}$.

We have the following theorem.

Theorem 1 For the CTP with the blockage sequence $E_{k}$, the competitive ratio of the greedy strategy is $2^{k+1}-1$.

Proof We denote the Greedy Strategy as GS. Let $O D$ denote the shortest path from $O$ to $D$ without any blockage in $G$. The traveller takes the shortest path $O D$ from the source $O$ first. When the traveller sees the blocked edge $e_{i}$, he/she takes the shortest path from $O_{i}$ to $D$ which does not make use of $e_{i}$. Let $\left(O_{0}, O_{1}, O_{2}, \ldots, O_{i}, O_{k-1}, O_{k}, O_{k+1}\right)$ denote the traversed path from $O$ to $D$ of the greedy strategy, where $O_{0}=O, O_{k+1}=D$, and let $C_{G S}\left(O_{i} O_{i+1}\right)$ denote the total cost from $O_{i}$ to $O_{i+1}$. The total cost of the GS from $O$ to $D$ satisfies

$$
\begin{aligned}
C_{G S}\left(O D \mid E_{k}\right) \leq & C_{o p t}(O D)+C_{G S}\left(O_{1} D \mid E_{1}\right) \\
& +C_{G S}\left(O_{2} D \mid E_{2}\right)+\cdots+C_{G S}\left(O_{k} D \mid E_{k}\right) .
\end{aligned}
$$

Note that $C_{G S}\left(O_{1} O_{2}\right), C_{G S}\left(O_{2} O_{3}\right), \ldots, C_{G S}\left(O_{k-1} O_{k}\right)$ are parts of $C_{G S}\left(O_{1} D \mid E_{1}\right)$, $C_{G S}\left(O_{2} D \mid E_{2}\right), \ldots, C_{G S}\left(O_{k-1} D \mid E_{k-1}\right)$, respectively.

Since

$$
\begin{gathered}
C_{G S}\left(O_{1} D \mid E_{1}\right) \leq C_{G S}\left(O O_{1}\right)+C_{o p t}\left(O D \mid E_{1}\right), \\
C_{G S}\left(O O_{1}\right) \leq C_{o p t}(O D) \leq C_{o p t}\left(O D \mid E_{1}\right),
\end{gathered}
$$

we have

$$
C_{G S}\left(O_{1} D \mid E_{1}\right) \leq 2 C_{o p t}\left(O D \mid E_{1}\right) \text {. }
$$

Due to the following inequalities

$$
\begin{aligned}
C_{G S}\left(O_{2} D \mid E_{2}\right) & \leq C_{G S}\left(O O_{1}\right)+C_{G S}\left(O_{1} O_{2}\right)+C_{o p t}\left(O D \mid E_{2}\right), \\
C_{G S}\left(O_{1} O_{2}\right) & \leq C_{G S}\left(O_{1} D \mid E_{1}\right) \leq 2 C_{o p t}\left(O D \mid E_{1}\right) \leq 2 C_{o p t}\left(O D \mid E_{2}\right), \\
C_{G S}\left(O O_{1}\right) & \leq C_{o p t}(O D) \leq C_{o p t}\left(O D \mid E_{1}\right) \leq C_{o p t}\left(O D \mid E_{2}\right),
\end{aligned}
$$

we have

$$
C_{G S}\left(O_{2} D \mid E_{2}\right) \leq 2^{2} C_{o p t}\left(O D \mid E_{2}\right) .
$$




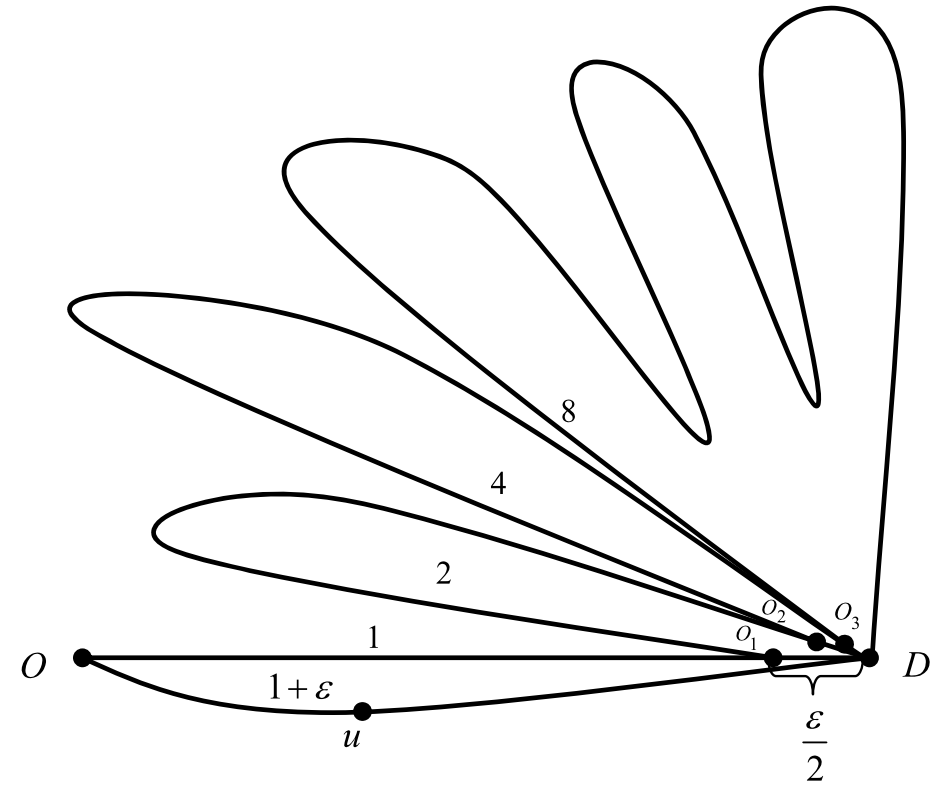

Fig. 1 Worst case of the GS

Similarly,

$$
\begin{aligned}
C_{G S}\left(O_{k} D \mid E_{k}\right) \leq & C_{G S}\left(O O_{1}\right)+C_{G S}\left(O_{1} O_{2}\right) \\
& +\cdots+C_{G S}\left(O_{k-1} O_{k}\right)+C_{o p t}\left(O D \mid E_{k}\right) \\
\leq & 2^{k} C_{o p t}\left(O D \mid E_{k}\right) .
\end{aligned}
$$

In summary, we have

$$
\begin{aligned}
C_{G S}\left(O D \mid E_{k}\right) \leq & C_{o p t}(O D)+2 C_{o p t}\left(O D \mid E_{1}\right) \\
& +2^{2} C_{o p t}\left(O D \mid E_{2}\right)+\cdots+2^{k} C_{o p t}\left(O D \mid E_{k}\right) \\
\leq & \left(2^{k+1}-1\right) C_{o p t}\left(O D \mid E_{k}\right) .
\end{aligned}
$$

This concludes the proof.

From the definition, the competitive ratio is a worst case performance measure. We present below an example illustrating the worst case scenario, thus showing that the above performance analysis for the greedy strategy is tight.

In Fig. $1, O D$ is the shortest path from $O$ to $D$ without any blockage in $G$ and $C_{\text {opt }}(O D)=1$. There is a path $(O, u, D)$ from $O$ to $D$, whose length is $1+\varepsilon$, where $\varepsilon$ is a positive number. No blockage will occur along $(O, u, D)$. Let the length of $O_{1} D, O_{2} D, \ldots, O_{k} D$ be $\frac{\varepsilon}{2^{k}}$. If the traveller knows the blocked edge $e_{1}$ and takes the path $\left(O_{1}, O, u, D\right)$, then he/she ends the trip and the total traversed cost is $2+\frac{\varepsilon}{2}$. However, following the greedy strategy the traveller chooses the shortest path from 
$O_{1}$ to $D$ instead of the path $\left(O_{1}, O, u, D\right)$ which does not make use of $e_{1}$. We have $C_{G S}\left(O_{1} D \mid E_{1}\right) \leq 2 \leq 2+\frac{\varepsilon}{2}$.

Similarly, we have

$$
\begin{aligned}
& C_{G S}\left(O_{2} D \mid E_{2}\right) \leq 4 \leq 4+\frac{\varepsilon}{4}, \\
& C_{G S}\left(O_{3} D \mid E_{3}\right) \leq 8 \leq 8+\frac{\varepsilon}{8}, \\
& \cdots \cdots \cdots \cdots \cdots \cdots \cdots \cdots \cdots \cdots \cdots \\
& C_{G S}\left(O_{k} D \mid E_{k}\right) \leq 2^{k} \leq 2^{k}+\frac{\varepsilon}{2^{k}} .
\end{aligned}
$$

Therefore the traveller takes the path $\left(O, O_{1}, O_{2}, \ldots, O_{i}, O_{k-1}, O_{k}, D\right)$. If $\varepsilon \rightarrow 0$, then we have the following corollary.

Corollary 1 For the CTP with the blockage sequence $E_{k}$, the competitive ratio of the greedy strategy is tight.

\subsection{The comparison strategy}

Comparison Strategy: When the traveller reaches $O_{i}$ and knows that the edge $e_{i}=$ $\left(O_{i}, Y_{i}\right)$ is blocked, he/she takes the greedy strategy or the reposition strategy according to the following condition. If $C_{G S}\left(O_{i} D \mid E_{i}\right) \leq C_{o p t}\left(O D \mid E_{i}\right)(i=1,2, \ldots, k)$, then the traveller takes the greedy strategy. If $C_{G S}\left(O_{i} D \mid E_{i}\right)>C_{o p t}\left(O D \mid E_{i}\right)$ $(i=1,2, \ldots, k)$, then the traveller takes the reposition strategy.

We have the following theorem regarding the comparison strategy.

Theorem 2 For the CTP with the blockage sequence $E_{k}$, the competitive ratio of the comparison strategy is $2 k+1$.

Proof We denote the Comparison Strategy as CS. Note that there are two types of subpaths in the traversed route $\left(O, O_{1}, O_{2}, \ldots, D\right)$. The first type is $\left(O, O_{1}, O_{2}, \ldots\right.$, $O_{j}$ ), where the traveller continuously uses the greedy strategy each time after observing the blocked edge (except the last one). In this case, for all $i \in\{1,2, \ldots, j\}$, it holds that

$$
C_{G S}\left(O_{i} D \mid E_{i}\right) \leq C_{o p t}\left(O D \mid E_{i}\right) .
$$

Then, the total cost of the greedy strategy part of the comparison strategy satisfies

$$
\begin{aligned}
C_{C S}\left(O D \mid E_{j-1}\right) \leq & C_{o p t}(O D)+C_{G S}\left(O_{1} D \mid E_{1}\right) \\
& +C_{G S}\left(O_{2} D \mid E_{2}\right)+\cdots+C_{G S}\left(O_{j-1} D \mid E_{j-1}\right) \\
\leq & C_{o p t}(O D)+C_{o p t}\left(O D \mid E_{1}\right)+C_{o p t}\left(O D \mid E_{2}\right) \\
& +\cdots+C_{o p t}\left(O D \mid E_{j-1}\right) .
\end{aligned}
$$


From Lemma 1, it follows that

$$
C_{C S}\left(O D \mid E_{j-1}\right) \leq j \cdot C_{o p t}\left(O D \mid E_{j-1}\right)
$$

The second type is $\left(O_{j}, O\right)$, i.e., after observing a blocked edge at $O_{j}$, the traveller returns to the source $O$. After each return trip to the source, the traveller can go along the shortest path from $O$ to $D$ on which the observed failed edges have been removed from $G$. The total traversed cost from $O$ to $O_{j+1}$ is

$$
\begin{aligned}
C_{C S}\left(O D \mid E_{j}\right) & =2 C_{C S}\left(O D \mid E_{j-1}\right)+C_{o p t}\left(O D \mid E_{j}\right) \\
& \leq 2 C_{C S}\left(O D \mid E_{k}\right)+C_{o p t}\left(O D \mid E_{k}\right) \\
& =(2 j+1) C_{o p t}\left(O D \mid E_{k}\right) .
\end{aligned}
$$

Similarly, the traveller continuously uses the greedy strategy after $O_{j}$ and returns to $O$ when he/she sees a blocked edge at $O_{j+i}, 1 \leq i \leq k-j$. We have

$$
\begin{aligned}
C_{C S}\left(O D \mid E_{j+i}\right) \leq & C_{C S}\left(O D \mid E_{j}\right)+(i-1) C_{o p t}\left(O D \mid E_{k}\right) \\
& +i \cdot C_{o p t}\left(O D \mid E_{k}\right)+C_{o p t}\left(O D \mid E_{k}\right) \\
\leq & (2 j+1) C_{o p t}\left(O D \mid E_{k}\right)+2 i \cdot C_{o p t}\left(O D \mid E_{k}\right) \\
= & (2(j+i)+1) C_{o p t}\left(O D \mid E_{k}\right) .
\end{aligned}
$$

From the above analysis, the total traversed cost from $O$ to $D$ is

$$
C_{C S}\left(O D \mid E_{k}\right)=(2 k+1) C_{o p t}\left(O D \mid E_{k}\right) .
$$

This concludes the proof of Theorem 2 .

\section{Application in an urban traffic environment}

From the analysis in Sect. 3, the competitive ratio of the greedy strategy is much larger than that of the reposition strategy in the worst case. However, in practice, why the greedy strategy and the comparison strategy are preferred by people in some situation, like driving in an urban traffic environment? In this section, we propose an explanation of why the greedy strategy and the comparison strategy are usually preferred by drivers in an urban traffic environment, based on an argument related to the length of the second-shortest path in a grid graph.

Let $C_{G S}\left(O_{i} D \mid E_{i-1}\right)$ denote the traversed cost from $O_{i}$ to the destination $D$ only when the traveller knows the blocked edge series $E_{i-1}=\left(e_{1}, e_{2}, \ldots, e_{i-1}\right)$. In addition, let

$$
\frac{C_{G S}\left(O_{i} D \mid E_{i}\right)}{C_{G S}\left(O_{i} D \mid E_{i-1}\right)}=\gamma_{i}
$$

for all $i$ and there exists a constant $\alpha$ such that $1 \leq \gamma_{i} \leq \alpha$. We call such a condition the pseudo-grid condition. And with that we have the following theorem. 
Theorem 3 For any greedy strategy GS satisfying the pseudo-grid condition, we have the following inequality

$$
C_{G S}\left(O D \mid E_{k}\right) \leq \alpha^{k} C_{o p t}\left(O D \mid E_{k}\right) .
$$

Proof Using the above definitions and Lemma 1, we can obtain that

$$
\begin{aligned}
& \frac{C_{G S}\left(O D \mid E_{k}\right)}{C_{o p t}\left(O D \mid E_{k}\right)} \\
& \leq \frac{C_{G S}\left(O D \mid E_{k-1}\right)-C_{G S}\left(O_{k} D \mid E_{k-1}\right)+C_{G S}\left(O_{k} D \mid E_{k}\right)}{C_{o p t}\left(O D \mid E_{k-1}\right)} \\
& \leq \frac{C_{G S}\left(O D \mid E_{k-1}\right)+(\alpha-1) C_{G S}\left(O_{k} D \mid E_{k-1}\right)}{C_{o p t}\left(O D \mid E_{k-1}\right)} \\
& \leq \frac{C_{G S}\left(O D \mid E_{k-1}\right)+(\alpha-1) C_{G S}\left(O D \mid E_{k-1}\right)}{C_{o p t}\left(O D \mid E_{k-1}\right)} \\
& =\frac{\alpha C_{G S}\left(O D \mid E_{k-1}\right)}{C_{o p t}\left(O D \mid E_{k-1}\right)} \\
& \quad \vdots \\
& \leq \frac{\alpha^{k} C_{G S}(O D)}{C_{o p t}(O D)} \\
& =\alpha^{k} .
\end{aligned}
$$

This concludes the proof of Theorem 3 .

If $\alpha$ is close to 1 , that is to say, the traversed cost of the second shortest path approaches the cost of the shortest path $O D$ (when there is no blockage in $G$ ), then the greedy strategy is obviously a better choice. In fact, as long as $\alpha<(2 k+1)^{1 / k}$, we can see that the performance measure of the greedy strategy is better than that of the reposition strategy. We next show that in a practical setting, namely, in an urban traffic environment the greedy strategy in fact outperforms the reposition strategy.

Let $G(V, E)$ denote an undirected planar grid network, there are $m+1$ rows of nodes in horizontal direction and $n+1$ columns of nodes in vertical direction. $V=\left\{v_{i j}\right\}$ denotes the nodes of $G(V, E), E\left\{e\left(v_{i j}, v_{i, j+1}\right) \cup e\left(v_{i j}, v_{i+1, j}\right)\right\}$ denotes a set of the edges between the two nodes, and $i=0,1,2, \ldots, m ; j=0,1,2, \ldots, n$. The passing time of each edge on $G(V, E)$ is 1 . The total number of blockages happening at $G(V, E)$ is no more than $k$, and $k<m \leq n$. We discuss two cases as follows.

Case 1. The source node and the destination node are on the same street.

Let $v_{00}$ denote the source node and $v_{0 n}$ denote the destination node. If there is no blockage happening in $G(V, E)$, the shortest path from $v_{00}$ to $v_{0 n}$ is $P\left(v_{00}, v_{01}, \ldots, v_{0 j}, \ldots, v_{0 n}\right)$ and the optimal travel time is $n$. In case of blockages, the traveller will choose an optimal path in $G$, with the blocked edges removed. It is 


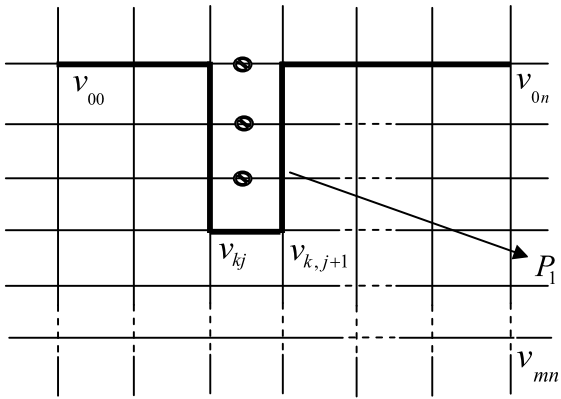

(a)

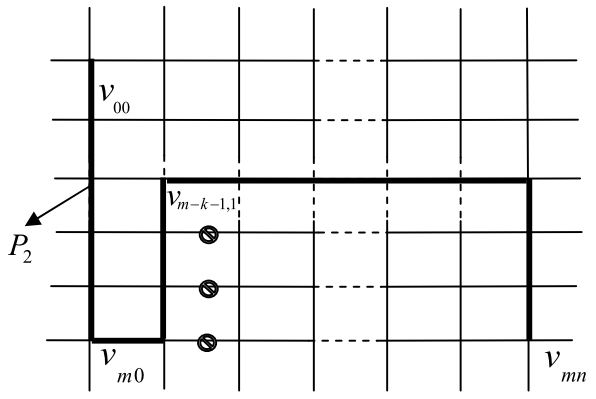

(b)

Fig. 2 Strategy application for a grid-type network

easy to see that when there is some blockage on $P$, then the optimal travel time is at least $n+2$.

Using the greedy strategy, the worst case occurs when the traveller sees $k$ blockages, i.e., $e\left(v_{00}, v_{01}\right), e\left(v_{10}, v_{11}\right), \ldots, e\left(v_{k-1,0}, v_{k-1,1}\right)$ are all blocked. Then the traveler first arrives at $v_{k 0}$, follows the path from $v_{k 0}$ to $v_{k 1}$ and finally arrives at $v_{0 n}$ without dealing with any blockage. The resulting path may look like $P_{1}$ in Fig. 2a and the travel time of $P_{1}$ is $2(k+1)+n$. The ratio of the travel time of the greedy strategy to the travel time of the optimal offline strategy is $\frac{n+2(k+1)}{n+2}$. If $k \ll m<n$, then $\frac{n+2(k+1)}{n+2} \longrightarrow 1$. In this case, the travel time of greedy strategy is close to the travel time of the optimal offline strategy. If $k \sim m \sim n$, then $\frac{n+2(k+1)}{n+2} \longrightarrow 3$, which is not too bad.

Case 2. The source node and the destination node are not on the same street.

Assume that the traveller wants to reach from the source $v_{00}$ to the destination $v_{m n}$. There are $\frac{(m+n) !}{m ! n !}$ shortest paths between the two vertices and the traveller can follow any of them. If there is no blockages happening on $G(V, E)$, then the travel time of each shortest path is $m+n$. If some blockages happen, the optimal travel time from $v_{00}$ to $v_{m n}$ must be at least $m+n$.

Again, using the greedy strategy, the worst case occurs as follows. When the traveller moves from $v_{00}$ to $v_{m 0}$, he/she sees no blockages at all. Then he/she arrives at $v_{m 1}$ and faces $k$ blockages. The resulting travel path is $P_{2}$, as shown in Fig. 2 b.

The travel time from $v_{00}$ to $v_{m n}$ is $n+m+2(k+1)$. The ratio of travel time of the greedy strategy to the travel time of the optimal offline strategy is $\frac{n+m+2(k+1)}{m+n}$. If $k \sim m \ll n$, then $\frac{3 m+n}{m+n} \longrightarrow 1$. If $k \sim m \sim n$, then $\frac{3 m+n}{m+n} \longrightarrow 2$. This is again acceptable in practice.

From the above analysis, the greedy strategy is in fact more reasonable under such a practical setting.

\section{Concluding remarks}

In this paper we propose and analyze two online strategies for the Canadian Traveller Problem. While the greedy strategy has the worst competitive ratio compared with 
the (known) reposition strategy and the comparison strategy, we show that under a special practical model the greedy strategy is a better choice.

Acknowledgements We gratefully acknowledge a number of valuable comments and suggestions given by the anonymous referees.

Open Access This article is distributed under the terms of the Creative Commons Attribution Noncommercial License which permits any noncommercial use, distribution, and reproduction in any medium, provided the original author(s) and source are credited.

\section{References}

Papadimitriou CH, Yannakakis M (1991) Shortest paths without a map. Theor Comput Sci 84(1):127-150 Bar-Noy A, Schieber B (1991) The Canadian traveller problem. In: Proceedings of the second annual ACM-SIAM symposium on discrete algorithms, pp 261-270

Westphal S (2008) A note on the $k$-Canadian traveler problem. Inf Process Lett 106(3):87-89

Zhu Z, Xu Y, Liu C (2003) Scheduling for on-line routing problem and its competitive strategy analysis. J Syst Eng 18(4):261-270. (In Chinese)

Hu M (2005) Comparison Strategy and its competitive ratio analysis of scheduling for on-line routing. J Ningxia Univ 26(3):207-210. (In Chinese)

Takimoto E, Warmuth M (2003) Path kernels and multiplicative updates. J Mach Learn Res 4(5):773-818

Kalai A, Vempala S (2005) Efficient algorithms for online decision problems. J Comput Syst Sci 71:291307

Blum A, Even-Dar E, Ligett K (2006) Routing without regret: on convergence to Nash equilibria of regret minimizing algorithms in routing games. In: Proceedings of the 25th annual ACM symposium on principles distributed computing, pp 45-52

Sleator D, Tarjan R (1985) Amortized efficiency of list update and paging rules. Commun ACM 28(2):202208

Borodin A, El-Yaniv R (1998) Online computation and competitive analysis. Cambridge University Press, Cambridge

Fiat A, Rabani Y, Ravid Y (1998) Competitive $k$-server algorithms. In: Proceedings of the 22nd IEEE symposium on foundation of computer science, pp 454-463

Fiat A, Woeginger GJ (1998) Online algorithms: The state of art. Springer, Berlin 